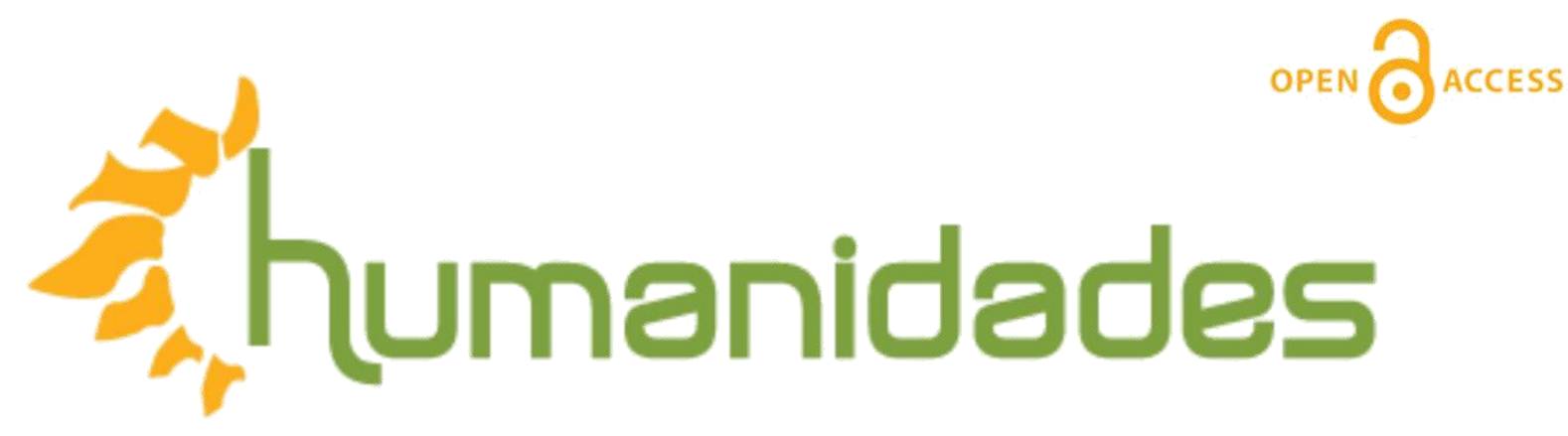

Revista de la Escuela de Estudios Generales, Universidad de Costa Rica

Julio-diciembre, 2017 • Volumen 7, número 2・EISSN 2215-3934•

Recibido: 15 - Enero - 2017 Aceptado: 15-Mayo-2017

\title{
Marqués de Sade: en contra del sexo-género determinista y la heterosexualidad excluyente
}

DOI: http://dx.doi.org/10.15517/h.v7i2.29577

\section{Andrés Solano-Fallas}

Universidad Estatal a Distancia, Escuela de Ciencias de la Educación. COSTA RICA.

Correo electrónico: sadsunsea@gmail.com

\footnotetext{
Todos los derechos reservados. Universidad de Costa Rica. Esta revista se encuentra licenciada con Creative Commons. Reconocimiento-NoComercial-SinObraDerivada 3.0 Costa Rica.

Correo electrónico: humanidades@ucr.ac.cr / Sitio web: http://revistas.ucr.ac.cr/index.php/humanidades
} 


\title{
Marqués de Sade: en contra del sexo-género determinista y la heterosexualidad excluyente
}

\section{Resumen}

Este trabajo analiza la postura filosófica en obras seleccionadas del Marqués de Sade en torno a la diversidad sexual. En un primer momento se estudia la crítica que lleva a cabo a la concepción determinista de sexo-género; y en un segundo momento, se examina su crítica a la concepción de una heterosexualidad obligatoria y normativa. En cada momento, se explican los supuestos filosóficos que le sirven de fundamento. Estas dos críticas de Sade permiten concebir cómo en su obra literaria y pensamiento la naturalidad de la diversidad sexual es explicada y posible. Cabe señalar que este trabajo se realiza desde un abordaje analítico.

Palabras claves: Marqués de Sade, diversidad sexual, sexo-género, heterosexualidad, naturaleza.

\section{Marquis de Sade: against the sex-gender determinism and the closed heterosexuality}

\begin{abstract}
This paper analyses the philosophical position of the Marquis de Sade's selected opera, on sexual diversity. In a first moment, it will be study the critic he carries on to the conception of sex-gender determinism. In a second moment, it will be examine his critic to the conception of an obligatory and normative heterosexuality. In each moment, it is explain the philosophical assumptions that serve him as basis. This two critics of Sade, allows conceiving how in his literary opera and thought the naturalness of sexual diversity it is explained and possible. It should be noted that this paper is from an analytical approach.
\end{abstract}

Keywords: Marquis de Sade, sexual diversity, sex-gender, heterosexuality, nature. 


\section{Introducción}

Este trabajo consiste en abordar la postura filosófica del Marqués de Sade, en torno a la diversidad sexual, por medio de sus críticas a dos concepciones, hoy conocidas como el determinismo de sexogénero (cf. Calvo, 2012; Laks-Eizirik en Alizade et al, 2004; y Molina-Petit, 1994) y la heterosexualidad obligatoria y normativa (cf. Rich en Navarro y Stimpson, 1999; y Butler, 2006). El abordaje se realiza de la siguiente manera. Se expone en qué trata la primera concepción, para ofrecer seguidamente la crítica del Marqués. De igual modo se procederá con la segunda concepción, para brindar la respectiva crítica. En la medida que se exponga cada crítica, se explican los fundamentos filosóficos a los cuales apela Sade para oponerse a tales concepciones. Esto servirá para ir mostrando cómo en el planteamiento del Marqués se manifiesta la diversidad sexual.

La postura filosófica de Sade, que se presenta en este trabajo, se hace con base en la siguiente selección de obras: Las 120 jornadas de Sodoma (1785), Filosofía en el Tocador (1795) y Notas a "Los días en Florbelle" (1806-1807 y quizá años siguientes) -de ahora en adelante: Las 120, F. Tocador y Notas-. Brevemente, la primera obra es un borrador y esquema que jamás finalizó debido a que perdió el manuscrito; en todo caso, narra las orgías que planearon cuatro amigos degenerados en el Castillo de Silling, las cuales van desde simples placeres no violentos hasta escalar en asesinatos. Para ello se arman de todo un sequito que tendrán distintas funciones durante los 120 días que tomará lugar. La segunda obra, escrita en forma teatral, narra la educación sexual-moral que la joven Eugenia recibe de parte de su amiga, el hermano de su amiga, y un conocido "bujarrón". Esta educación se suponía durar dos días, pero solo acaece en uno, en el Tocador de la amiga. Y la tercera "obra", más bien, es un conjunto de notas escritas por el propio Marqués para pulir "Los días en Florbelle", empero fue quemada por su hijo menor para ahorrarle vergüenza a la familia, dado su alto contenido sexual y violento.

Finalmente, cabe comentar que, a excepción de $F$. Tocador, este trabajo entraña una complejidad y esfuerzo interpretativo de la filosofía de Sade, dado que las otras dos obras son versiones no acabadas. Las 120, si bien contiene la trama narrativa, una parte es un borrador y la otra solamente un esbozo de lo que sería el posterior desarrollo; mientras que las Notas no constituyen la obra, sino precisamente eso, a saber, unos apuntes que constituyen en sí una aproximación de segundo orden a una obra que igualmente estaba en su fase de redacción, pero de la cual no se puede cotejar debido a su quema.

\section{Concepción determinista de sexo-género}

Esta concepción parte del supuesto de que existen elementos de la naturaleza humana que no solamente son fijos e inmutables, sino que constituyen un asidero para definir lo que socialmente se esperaría de cada persona, por lo que el género de cada persona, y sus distintos roles, estarían marcados por su sexo. Es decir, se establece una correspondencia unívoca y unidireccional entre sexo y género, siendo lo primero que dotaría de sentido a lo segundo, mientras lo segundo reconfirmaría y reproduciría al primero. Esta correspondencia conlleva a que todo aquello que no encaje a su parámetro sea considerado como incorrecto o monstruoso, según la escala de valores del grupo social en cuestión. Consiguientemente, no existe la posibilidad de siquiera pensar en la diversidad sexual o de género, porque el género no puede ser diverso ya que su asidero (el sexo) es estático. 
Aunado a lo anterior, esta concepción se entronca con un patriarcalismo, en la que asignación de roles y expectativas a cada persona se realiza con base en su sexo-género, según una escala valorativa que concibe al hombre como superior y modelo de la naturaleza humana (cf. Molina-Petit, 1994). Como señala Calvo (2013, p. 45), "[e]l hombre, desde muy pronto en la historia, se atribuyó a sí mismo todas las cualidades positivas y concedió las negativas a la mujer”. De esta manera, el sexo-género no solamente es fijo y terminante en el comportamiento social, sino que además cataloga y jerarquiza a las personas, colocando a un grupo mayor sobre otro grupo mayor, y por ende, creando seres superiores e inferiores. A razón de esto, jamás podría haber igualdad entre hombres y mujeres, porque se considera que la naturaleza ha dictaminado y creado un orden que no puede ser franqueado.

A pesar de que el determinismo de sexo-género se puede remontar a varios siglos atrás, conviene detenerse momentáneamente en la época que circunscribe al Marqués, y particularmente en dos campos que tenían fuerte incidencia, a saber, la filosofía y la medicina, en orden a ofrecer un mejor contexto de su crítica.

En el campo filosófico europeo, "la línea de pensamiento general relega sistemáticamente lo femenino" (Cortina en Fisas, 1998, p. 33), y se podría añadir, que lo hace con un fuerte machismo. Muestras de esto se puede encontrar en Lo bello y lo sublime de Kant, para quien una mujer debe mantenerse en lo que le es propio a su ser, y no inmiscuirse en asuntos de hombres, ya que "[e]l estudio trabajoso y la reflexión penosa, aunque una mujer fuese lejos en ello, borran los méritos peculiares de su sexo" (Kant, 1932, p. 38). Particularmente en Francia, se tiene a Rousseau que, de entre sus varias obras, el Emilio sobresale por su fuerte misoginia. Para el Ginebrino, la mujer debe ser un ser sumiso porque así lo ha querido la naturaleza, por cuanto que a los hombres los ha hecho fuerte y dominantes (cf. Rousseau, 1985). Cada uno tiene sus cualidades, por lo que se debe velar en cultivarlas, pero sin mezclarlas. Si el hombre cultiva sus cualidades en las mujeres, estaría obrando en perjuicio suyo, porque la mujer está para servirle, aclarando que "[n]o se trata de hacerles penosa su dependencia, basta con hacérsela sentir” (Rousseau, 1985, p. 427).

En el campo médico, el discurso que se aportaba para justificar el determinismo sexo-genérico, tenía la peculiaridad que se arrojaba a sí mismo "objetividad", por basarse en investigaciones "empíricas". Presentaba a las mujeres como seres que estaban listos para criar hijos, debido a la "combinación de la debilidad mental y muscular y la sensibilidad emocional" (Hunt en Ariés y Duby, 2005, p. 49) que supuestamente las caracteriza. Este discurso que sirvió para validar las diferencias sexo-genéricas, tuvo su epicentro con en el tratado Du systèm moral et physique de la femme (1775 y 1783) del médico francés Pierre Roussel, dado que obtuvo gran acogida, y básicamente reproducido por otros médicos.

De este modo se puede observar cómo el determinismo de sexo-género se proyectaba y reproducía en la época del Marqués, aludiendo a bases filosóficas y médicas que gozaban de estima. Lo problemático de esta concepción, yace en el cierre de posibilidades de una diversidad de sexogéneros, y en la jerarquización inferiorizante a la que eran sometidas las personas.

\section{Crítica al determinismo sexual-genérico}

Ante esta concepción, Sade la arremete en varias de sus obras, de las cuales se han seleccionado las Notas, Los 120 y F. Tocador. De manera general, se puede señalar que el Marques, si bien parte de que existen elementos sexuales que caracterizan a las personas, no considera que, en primer lugar, estos sean determinantes ni restrictivos, y consecuentemente que no "aprisionan" el género; y en 
segundo lugar, que no conducen a la subordinación sumisa de unos seres a otros por el solo hecho de tener ciertas diferencias sexuales.

En lo que respecta a Las 120 es en sí mismo una muestra de lo anterior, por cuanto que sus personajes principales, los cuatro amigos degenerados y las cuatro narradoras, son ubicados respectivamente en sus sexos, macho y hembra. Pero con la particularidad de que su perversión y lujuria no se debe en modo alguno por pertenecer a un sexo y no al otro. Tanto hombres como mujeres libertinos o nolibertinos no están restringidos a comportarse de determinada manera en relación a sus aspectos sexuales, sino según la constitución con que los dotó la naturaleza, pero sin prestar atención en quien sería malo o bueno.

Contrario a la concepción determinista que sobreestima el sexo, para el Marqués en Las 120 es una característica más de la persona. Ejemplos sobran en las dos descripciones que hace de los personajes de la novela - una versión larga y detallada, otra más resumida y concisa-, cuando describe a sus personajes principales, en la que muestra que el sexo, aunque condicione el rango de actuación para algunas actividades, no es el único rasgo definitorio de la persona: entre los machos, hay unos que poseen penes más largos o cortos, unos más vigorosos o ridículos, cuerpos más fornidos o más "afeminados"; mientras que entre las hembras hay unas que tienen pechos y otras no, otras un clítoris más grande $u$ otras no.

Sirva lo siguiente como muestra, dado que la lista es bastante amplia. A Durcet se le describe como "pequeño, bajo y rechoncho, pero su cuerpo es fresco, hermoso y blanco, semejante al de una mujer, y posee todos sus gustos; privado de darles placer, por lo ridículo de su tamaño, la ha imitado y se hace encular a cada instante del día" (Sade, 2009c, p. 71); mientras que al Duque se dice que está "dotado de un miembro gigantesco y de una fuerza prodigiosa" (Sade, 2009c, p. 70). Nótese como el cuerpo de Durcet es esbozado básicamente como el de una hembra, sin que esto atente su percepción como hombre, y a su vez, como el Duque es sobredotado con órganos sexuales cuya intención es manifestar su fuerza sexual, pero no caracterizarlo como más hombre que Durcet. Algo similar puede decirse de las mujeres. La Champville, una de las historiadoras, es caracterizada con un "clítoris largo y cosquilloso" (Sade, 2009c, p.72); mientras que a la Fachon, una de las criadas, "un chancro le devora la vagina, tiene un muslo quemado y un cáncer le roe el pecho" (Sade, 2009c, p. 74). Con estas dos mujeres, puede verse que cada una continua siendo concebida como tal (mujeres), independientemente de que una posea un clítoris más largo, y la otra esté perdiendo rasgos sexuales por enfermedades. Cada uno es identificado como hombre o mujer, pero sin hacer del sexo un elemento determinante.

Si el sexo fuese determinante, sería difícil de explicar cómo las víctimas, que son tanto hombres como mujeres de edad muy joven, no comparten el mismo carácter que los amigos depravados y las historiadoras y criadas; ya que en la síntesis que realiza el Marqués habla de lo bello que son las ocho muchachas, y de que "[n]inguna pluma es capaz de pintar las gracias, los secretos encantos" de los ocho muchachos (Sade, 2009c, p. 75), es decir, hace referencia a sus rasgos sexuales y otros, pero sin convertir el sexo en un elemento obligue a cada uno al seguimiento de un tipo de conducta y rol.

Las singularidades sexuales que presentan los seres humanos no conducen a que una persona sea más o menos hombre o mujer, por lo que en Sade la relación entre sexo y género no es unidireccional, sino dos grandes aspectos (entre otros más) que confluyen en la persona. Esta diferenciación es manifiesta en la escena en que los amigos degenerados conciben la idea de que las víctimas intercambien roles: "todas las muchachas de marinero y todos los muchachos de modistillas. El efecto que eso produjo fue el deseado: pocas cosas podían haberlos calentado más que el 
intercambio de géneros" (Sade, 2009c, p. 146). Nótese como sutilmente, el Marqués separa el sexo y el género: el sexo es algo que nace con la persona, por lo que no se puede obviar el estar sexuado, pero lo sexuado no es un impedimento para que las personas puedan asumir distintos géneros.

En el caso de F. Tocador, uno de los personajes principales, la Sra. De Saint-Ange, le explica a Eugenia que la mujer no está forzada a cumplir las funciones que el himeneo le atribuye en razón de su sexo: "El destino de la mujer es igual al de la loba, al de la perra: debe entregarse a todos los que la deseen. Encadenar a las mujeres por el absurdo lazo de un himeneo solitario entrañaría un ultraje evidente al destino que la naturaleza ha impuesto a las mujeres" (Sade, 2003a, p. 51). Si bien la expresión respecto del destino de la mujer puede ser fuerte para algunos lectores, ha de recordarse que es en aras del carácter del personaje; debido a que lo que interesa es el contenido que el Marqués está explicando, a saber, que la mujer no ha nacido para ser exclusivamente esposa y madre, y por ende, no ser dueña de sí mismo. Aunque posea la capacidad reproductiva, la naturaleza no la ha creado para que se dedique únicamente a ella, por cuanto que también la ha hecho para que disfrute de su cuerpo como bien le parezca, "tu cuerpo de te pertenece solo a ti, y solo tú tienes el derecho de gozarlo y hacer gozar con él a quien se te antoje" (Sade, 2003a, p. 53). De este modo, la reproducción (que es una característica sexual), no conduce a que los rasgos definitorios del género de una mujer deban necesariamente incluir la maternidad y la conyugalidad, como lo suponía el discurso filosófico y médico de la época.

Luego, en las Notas, deja entrever en algunas notas concernientes al personaje de Émilie de Valrose que el género de una persona no está supeditado inexorablemente al sexo. En el caso de Émilie, se eliminan todas las cualidades que se le suponían propias a su sexo, a saber, ese amor a la familia y sumisión a la misma. Ella no está obligada a tener que amar a su familia, como tampoco a tener que desear conformar una, por el mero hecho de ser mujer, como lo suponía el discurso médico. De hecho, este personaje es asesino y rencoroso: respecto de su madre, a quien odia profundamente, "[s] he has regretted having only one mother, and would have liked to sacrifice two of them" (cf. Nota \#71, Sade, 2003c, p. 100), pero sin ser presentado como una anomalía, sino como un producto más de la naturaleza. Puede notarse que en la nota \#71, a pesar de su brevedad, es indicativa de que el Marqués se opone a una concepción de mujer entregada a la familia. Siguiendo a Seifert (2012), atacar la figura de la madre en el pensamiento de Sade era fundamental en su obra para cuestionar el ámbito doméstico, ya que este constituía un mecanismo para reproducir valores epocales, tales como la sumisión de la mujer en aras de un amor a la familia. Si bien Sade en modo alguno está proponiendo que se deba matar a la madre para acabar con concepciones deterministas de sexo-género, lo que interesa es que en el fondo se hace evidente que el odio materno con el cual permea a su personaje, es una manera por la cual cuestiona los supuestos rasgos naturales que debe seguir una mujer.

Por otra parte, de manera similar a las obras anteriores -aunque con mayor semejanza a Las 120-, los personajes buenos y malos no están catalogados con base en su sexo, sino por las inclinaciones que recibieron de la naturaleza, según se expresa en la nota \#99: "one must never resist the inclinations that one has received from nature" (Sade, 2003c, p. 108). A pesar del carácter fragmentario de la notas, se puede extraer, por ejemplo, lo siguiente: en la nota \#99 se tiene a un monje que causó una catástrofe; en la nota \# 103 se menciona a uno de los personajes principales, Valrose, quien ha matado a varios miembros de su familia; en la nota \#70, a Senaport, otro personaje importante, que cometió incesto con su hermana, engendrando a Émilie; e incluso la misma Émilie quien termina matando a su madre mediante una tortura china, según la nota \#71. Nótese que los personajes mencionados en las notas actúan acorde a sus inclinaciones, según se establece en la nota \#99, sin que el sexo sea un determinante. De lo contrario, se vuelve al mismo problema antes señalado en Las 120, a saber, cómo explicar la existencia de víctimas, si estas comparten el mismo sexo que 
sus verdugos y asesinos. De este modo, la caracterización que Sade realiza de sus personajes (principalmente los malos), es un medio por el cual socaba el determinismo, cuestionando que los roles que asumen sus personajes no están condicionados por el sexo. Nuevamente, parte del hecho de que son sexuados, pero sin que se sujete con alguna expectativa reductivista de comportamiento e identificación.

Por tanto, debido a que el sexo es un rasgo entre otros, esto le permite al Marqués elevarse contra la concepción determinista al señalar que una persona, independientemente de su sexo, puede asumir el género que supone contrario. En la nota \#9 (Sade, 2003c, p.85) indica que "Émilie works very well as man and and (sic) I must take her through all the same situations as Valrose, sometimes as a man, sometimes as a woman". Obsérvese que Sade no está refiriéndose a una cuestión de simplemente vestir a una persona, sino que esa persona puede desarrollarse plenamente en el género opuesto, independientemente del sexo que posea, porque no hay ninguna ligadura sexual que se lo impida. Si la concepción determinista, tanto en su variante filosófica como médica, fuese cierta, situaciones como la presente no solamente no tendrían sentido, sino que además no podrían ocurrir y ni siquiera pensarse, por cuanto que la determinación del sexo sobre el género no debería permitir siquiera la posibilidad imaginativa.

Se puede notar que la postura del Marqués recurre a lo "empírico", tal y como lo hace la concepción determinista. Sin embargo, él ve -y aporta múltiples ejemplos, principalmente en Las 120 y F.Tocador- de que la naturaleza, si bien crea y ubica a las personas en dos grandes y amplias categorías, a saber, machos y hembras, no se sigue que el sexo predetermine el comportamiento y el género de la persona. Si así fuese, cabría esperar el mismo actuar en cada grupo sexual, empero no sucede, porque cada persona actúa según le impongan sus fuerzas y voluntad, de acorde a las posibilidades que le permite su constitución sexual, tal como lo expresa la Sra. De Saint-Ange a Eugenia: "no debe haber límites para tus placeres, salvo los que impongan tus fuerzas y tu voluntad" (Sade, 2003a, 52). Por tanto, el sexo en Sade es la base sobre la cual empieza a operar una persona, pero a la que no se ve reducida su existencia. Mientras en la concepción determinista el género no es más que una derivación o profundización de características sexuales; Sade concibe el sexo como una parte complementaria, que se relacionará con el género en la medida que la persona madure, creando una relación en ambas direcciones que se influirán mutuamente.

Aunado a lo anterior, el sexo al no determinar el género de una persona, tampoco se convierte en categoría subordinativa. Para él, no tiene sentido que una persona sea sometida a otra de manera automática en razón de su diferenciación sexual, debido al simple hecho de que todos son productos de la naturaleza: "nunca se puede otorgar derecho legítimo a uno de los sexos de apoderarse exclusivamente del otro" (Sade, 2003a, 163). Si un ser somete a otro -como ocurre en Las 120 y en las Notas-, lo puede hacer si así lo desea, pero deberá valerse de su fuerza y/o astucia, pero no alegando a que la naturaleza lo ha predispuesto de esa manera. De esta manera, Sade no solamente rechaza a la concepción determinista, sino que puede decirse que le planteó serias dificultades, debido a que él está fundamentando su postura en lo "objetivo", es decir, la naturaleza misma: a esa a la que el pensamiento tradicional pretende aludir y que el discurso médico supone esclarecer. Siguiendo a Bloch (2013) y a Álvarez (1972), debe tenerse en cuenta que si bien los personajes de Sade son ficticios, estos están inspirados en la vivencia de sujetos reales que en modo alguno eran unos cuantos casos aislados. La existencia de sus personajes es sintomática de que la concepción determinista está planteando una postura que no se apega a la realidad: ni en su discurso filosófico que no hace más aludir a opiniones que no tienen asidero "objetivo", como tampoco en el discurso médico que ignora o minimiza lo que la realidad le presenta. En Sade lo "empírico" (la naturaleza) no conduce a ningún determinismo sexual-genérico ni subordinativo. 


\section{Concepción de la heterosexualidad obligatoria y normativa}

Esta concepción asume que existe un vínculo inquebrantable entre el sexo-género de una persona y su orientación sexual, el cual supone que cada sexo-género tiene una predisposición sexual a su contrario, siendo por consiguiente la heterosexualidad el modelo por excelencia. Esto se debe a que el sexo, al ser considerado la supuesta base natural del género y de otras características, la orientación sexual era una más de esas características, lo que causaba que la heterosexualidad fuese revestida de naturalidad, mientras que cualquier otra orientación sexual fuese denigrada y catalogada de inmoral. Consiguientemente, para esta concepción, ser heterosexual era una obligación de la cual nadie debía atentar ni rehusar. Sin embargo, no era una heterosexualidad que gozase de amplia libertad. Por el contrario, estaba marcada por una serie de pautas -algunas más específicas que otras, dependiendo del momento histórico-, cuya finalidad era normar que tipos de actos sexuales heterosexuales eran permitidos, y por ende, ser calificados de "heterosexuales". Badinter (1993) explica que el sodomita no era exclusivamente aquel hombre que sostuviese relaciones sexuales con otro hombre, sino también aquel que mantuviese sexo anal con mujeres. Por lo que un hombre, si bien puede tener una orientación por mujeres, esta concepción le dicta que debe limitarse a aquellas prácticas sexuales consideradas correctas, debido a que la naturaleza impone lo que se supone permitido. A lo sumo, una persona podía decidir no ejercer prácticas heterosexuales, sin que esto implicase una violación a la naturaleza, como sería el caso de sacerdotes y monjas. Lo que interesaba era que si no practicaba su heterosexualidad, no incurriese en otras "ilícitas" y "antinaturales". De este modo, la heterosexualidad estaba normada, por lo que no bastaba que el objeto de deseo de una persona fuese una de su sexo-género contrario, sino que era necesario, para considerarse tal, una observancia y cuido en lo que realizase.

\section{Crítica a la heterosexualidad obligatoria y normativa}

La crítica que el Marqués realiza a la concepción restrictiva e ineludible de la heterosexualidad, consta de dos puntos principales. El primero consiste en la eliminación de la causalidad entre perversión y orientaciones sexuales no-heterosexuales, sin eliminar la naturalidad de la perversión; y el segundo en la desvinculación entre sexo-género y orientación sexual.

El primer punto principal lo lleva a cabo mediante la puesta en escena de sus personajes, los cuales van desde heterosexuales hasta homosexuales, pasando por bisexuales. Él no se limita a hacer únicamente una referencia o una detallada descripción de sus pasiones sensoriales, sino que también ofrece rasgos fisiológicos y de actitud, entre otras. En Las 120, y muy posiblemente en las Notas, ofrece una mayor precisión; mientras que en $F$. Tocador, al estar escrita como obra teatral, los rasgos se van conociendo a medida que se desenvuelven las escenas. A la hora de hacer esto, Sade se cuida de no asociar causalmente las orientaciones sexuales no-heterosexuales a lo perverso, y de catalogarlas de antinatural, como pretende la concepción obligatoria de la heterosexualidad. En Las 120, sin duda alguna, los cuatro amigos degenerados son unos seres perversos, empero, dicha perversidad no se debe a que sean homosexuales o bisexuales. En este caso, la perversidad sería una característica de su personalidad que les induce a causar daños y buscar placeres a como dé lugar, independientemente de su orientación sexual. De modo similar sucede en F.Tocador, con la singularidad de que Eugenia recibe una educación sobre el libertinaje, el cual no es enfocado como una aberración de la naturaleza, evitando seguir el argumento causal de la heterosexualidad obligatoria, aludiendo (mediante Dolmancé) que si la naturaleza hubiese considerado incorrecto prácticas libertinas, como el sexo anal, no las hubiese permitido en primer lugar: " $[s] i$ no hubiese sido su intención que penetrásemos culos, ¿habría hecho tan proporcionado su orificio a nuestros 
miembros" (Dolmancé al Caballero de Mistival; Sade, 2003a, p. 103). Esta consideración será una constante, por cuanto que en las Notas, por lo que se puede inferir, la orientación sexual no es causante de perversión de los personajes, ni mucho menos tomada como algo antinatural.

Por tanto, el primer punto de su crítica radica en la abolición de lo "antífisico" (cf. Roudinesco, 2009), es decir, eliminar cualquier etiqueta de perversidad o antinaturalidad a toda orientación noheterosexual. La concepción obligatoria y normativa de la heterosexualidad se ha concentrado únicamente en defender lo que ha querido valorar como correcto, aludiendo a que existe un orden en la naturaleza que ha reglamentado que cualquier orientación sexual que no fuese heterosexual es una perversión a sus leyes, y por ende aberrante, antinatural. En las obras seleccionadas, se puede apreciar que las descripciones de sus personajes aluden a lo "objetivo", a saber, la naturaleza. Esta es nuevamente el sustento teórico de Sade, ya que ella sería el "actor intelectual y material" de lo existente, y por derivación, de las orientaciones sexuales. Para el Marqués no tiene sentido decir, desde dicha concepción de la heterosexualidad, que un acto sexual es antinatural y considerarlo como algo degradado a la norma natural, debido a que la naturaleza se estaría degradando a sí misma al permitir y producir algo "degradado", según se expresa mediante Dolmancé, cuando este le dice a Eugenia: en relación a la penetración anal, "[e]s absurdo decir que esta manera la ultraja. ¿Cómo podría ser así, es la naturaleza la que nos inspira? ¿Acaso puede ella disponer algo que la degrade?” (Sade, 2003a, p.64). De este modo, aplica lógica básica al cuestionar por qué si algo no quiere o no permite ciertas cosas "inadecuadas", aún así accede a hacerlas, y no busca los medios para eliminarlas.

Aunado a lo anterior, como se indició, el Marqués con esta crítica elimina la vinculación causal entre perversión y orientaciones sexuales no-heterosexuales. Se puede considerar que Sade concibe que la perversión y la orientación sexual sean como dos "elementos", entre muchos otros, que la naturaleza utiliza a la hora de realizar sus creaciones, por lo que no existe razón suficiente que obligue que haya algunas orientaciones sexuales que sean indefectiblemente perversas. Por ejemplo, los ocho "enculadores" de Las 120, a pesar de que terminan siendo cómplices más o menos pasivos de las crueldades de los cuatro amigos degenerados, no son caracterizados como perversos por el hecho de vender sus anos y otros servicios sexuales. En la versión larga descriptiva de los personajes de la novela, se indica que su elección para participar en las jornadas orgiásticas se basó en que tuvieran una edad razonable, que fuesen guapos y con penes bastante grandes, por lo que "bastaba pagarles el viaje, el trabajo, y se volvían a casa" (Sade, 2009c, p. 47). Por lo que se describe de ellos, son homosexuales pagados para atender los deseos de los amigos; no se les contrata porque fuesen perversos, por lo que no se establece una relación entre homosexualidad y perversidad. También podría tomarse como indicativo de esto, las descripciones que realiza de las historiadoras y las criadas. Por ejemplo, Marie, una de las criadas, se dice que "[h] a parido y matado a catorce criaturas" (Sade, 2009c, p. 73); de la Desgranges, que "[t]odo en ella delata la inclinación al crimen"; y la Martiene, quien es "solo ha conocido el placer de Sodoma" (Sade, 2009c, p. 73) Básicamente son mujeres heterosexuales que practican el sexo anal, sin que se les describa que a causa de dichos gustos anales es que son perversas. Sino que su perversidad proviene de la propia naturaleza que las hizo de dicha manera, con independencia de sus gustos sexuales. Sade insiste, mediante sus personajes, que la heterosexualidad obligada erra al valorar lo no-heterosexual como necesariamente perverso en orden a descalificarlo. Lo perverso existe y no se le puede negar, porque es producto de la naturaleza. Pero la naturaleza no lo atribuyó exclusivamente a las orientaciones noheterosexuales.

El segundo punto principal de su crítica yace en que no existe una vinculación entre sexo-género y orientación sexual. Esta falta de vinculación no es descrita en Sade como una anomalía, sino 
considerada con un tono de naturalidad, según se puede observar representada en sus personajes. Ninguno de ellos tiene una orientación sexual específica por el mero hecho de su sexo-género. En las tres obras seleccionadas hay tantos hombres como mujeres bisexuales y homosexuales, y en menor medida heterosexuales. La consecuencia inmediata -y perjudicial para la concepción de la heterosexualidad obligatoria- es que se elimina cualquier normatividad. En primer lugar, por lo que se indicó en el primer punto principal, las orientaciones no-heterosexuales son naturales, por lo que no existe ningún mandato natural que conduzca a que las personas sean heterosexuales. Así como todas las preferencias sexuales son naturales, también lo será toda práctica sexual que se derive de estas, lo que plantea nuevamente que no existe ninguna disposición o serie de preceptos que indiquen cómo debe comportarse sexualmente una persona. En Las 120 indica, por medio de Durcet, que la persona debe guiarse por los sentidos: "son los únicos que deben guiarnos en todas las acciones de la vida, porque son los únicos cuyo órgano es realmente imperioso" (Sade, 2009c, p. 229). Asimismo, en las Notas, apunta que toda persona debe seguir sus inclinaciones, y no oponerse a ellas: "one must never resist the inclinations that one has received from nature" (Nota \#99, Sade, 2003c, p.108). Se puede inferir que no solamente no existen límites, sino que carece de sentido restringir las prácticas sexuales que la misma naturaleza ha creado. Otra vez, Sade apela a la lógica básica para atacar a la concepción de la heterosexualidad obligatoria y normativa, por cuanto que no se puede explicar por qué algo dispondría de unas pautas y censuraría otras, cuando pudo ahorrarse el trabajo de la censura mediante su eliminación.

En lo que respecta específicamente a la heterosexualidad, en F. Tocador, objeta la norma de la procreación. El Marqués no niega que la procreación sea un asunto natural, por cuanto que ha sido confeccionada por la naturaleza. Sin embargo, le resta sobreestimación, señalando que así como la naturaleza crea, también destruye. En razón de esto, no tiene sentido que solo se acepte como acto sexual válido -y por tanto, heterosexual- el coito con fines reproductivos, en razón de la capacidad reproductiva de los sexos. De las varias maneras de destruir, en la práctica heterosexual estaría la la masturbación, o la descarga del semen en otras cavidades, las cuales son igualmente aceptadas por la naturaleza, porque permite que ocurran: "es falso que la naturaleza pretenda que ese licor espermático esté absolutamente destinado a la procreación; si fuese así, no solo no permitiría que este derramamiento se produje en todos los casos [...], y de inmediato se opondría a que esas pérdidas se produjeran sin que haya coito, como ocurre durante el sueño o el recuerdo" (Sade, 2003a, p. 114). Lo que Sade estaría expresando, es que aún cuando la heterosexualidad no es la única orientación sexual -ni tiene por qué serlo-, la concepción heterosexual es muy reduccionista y restrictiva. De este modo, ni en la misma orientación heterosexual existen normas que deban ser respetadas, con base supuestamente en las capacidades sexuales de una persona.

\section{Conclusión}

En la obra literaria y en el pensamiento del Marqués, existe un fuerte rechazo a toda concepción que limite y restringa la diversidad sexual, tanto la que convierte en monolitos el sexo-género como la que excluye otras orientaciones sexuales. En Sade, la clave de sus críticas a las concepciones anteriores, recaen en el argumento principal y esencial del materialismo francés, a saber, la Naturaleza. Esta ha sido, es y será la productora de todo cuanto exista. Sin embargo, su producción es variada, sin que se privilegie ninguna manera en específico sobre otras formas. Precisamente este punto es lo que el Marqués critica a las concepciones anteriores, debido a que pretenden partir de un naturalismo, que no toma en cuenta la diversidad que se lleva a cabo gracias al poder genésico y destructivo de la Naturaleza, cayendo en un reductivismo sexual. 
En lo que respecta a la concepción determinista del sexo-género, como se indicó, Sade no niega que existan elementos sexuales que definan fisiológicamente a la persona. En este punto estaría de acuerdo con tal concepción, empero, difiere en el tanto en que tal concepción solidifica el sexo, y hace que todo lo que se le vincule, deba serlo de manera unidireccional y en relación causa-efecto; y que además, sea tomado como hecho "empírico" para subyugar sistemáticamente a las mujeres en razón de su diferencia sexual. De manera similar sucede con la heterosexualidad. El Marqués no niega que exista, pero critica que una falta de comprensión a la naturaleza conduce a considerar que existen, por un lado, reglas dentro de las prácticas heterosexuales, y por otro, que las orientaciones no-heterosexuales son perversiones y antinaturales, y no van acorde al sexo-género.

Por el contrario, los únicos límites que existen son los que cada persona se da a sí mismo, según las inclinaciones con que la naturaleza lo produjo. Podría considerarse que sus críticas son un llamado a vivir una diversidad sexual plena, en la que enfatiza que no existe $l o$ correcto y lo incorrecto "objetivo", ni la sumisión de un sexo-género a otro, y por ende libre de pseudo-normas supuestamente "naturales", en la que efectivamente la persona es un ser con ciertas características y orientaciones sexuales, pero sin estar atrapadas en ellas; por cuanto que en última instancia eso que lo hacen las concepciones deterministas del sexo-género y la heterosexualidad excluyente: aprisionar a la persona en su propio cuerpo. 


\section{Referencias}

Álvarez, A. J. (1972). Sade y el sadismo. México: Grijalbo.

Amorós, C. (1997). Tiempo de feminismo. Sobre feminismo, proyecto ilustrado y postmodernidad. Madrid: Ediciones Cátedra-Universitat de València-Instituto de la Mujer.

Badinter, E. (1993). XY. La identidad masculina. Madrid: Alianza.

Bloch, I. (2013). Marquis de Sade. The Man and His Age. Studies in the History of the Culture and Morals of the Eighteenth Century. Lexington: Magnolia Books.

Butler, J. (2006). Deshacer el género. Barcelona: Paidós.

Castañeda, M. (1999). La experiencia homosexual. Para comprender la homosexualidad desde adentro y desde afuera. Barcelona: Paidós.

Cavana, M. L. (2000). "Diferencia" en Amorós, C. (directora). 10 palabras clave sobre Mujer. Navarra: Verbo Divino.

Cobo-Bedia, R. (2000). "Género" en Amorós, C. (directora). 10 palabras clave sobre Mujer. Navarra: Verbo Divino.

Cortina, A. (1998). "El poder comunicativo. Una propuesta intersexual frente a la violencia" en Fisas, V. (ed). El sexo de la violencia. Género y cultura de la violencia. Barcelona: Icaria.

De la Mettrie, J. O. (1983). Obra Filosófica. Madrid: Editora Nacional.

D’Holbach, P. H. T., Barón (2008). Sistema de la naturaleza. Barcelona: Laetoli.

Dukes, H. (2013). Charla "Sexualidad y Género", en el marco de la Feria del Sexo y el Afecto, organizada el 12 de setiembre, por el Servicio de Psicología de la Coordinación de Vida Estudiantil de la Sede del Pacífico, Universidad de Costa Rica. [La charlista es miembro del Cuerpo de Paz Costa Rica]

Fernández-Alemany, M., y Sciolla, A. (1999). Mariquitas y marimachos. Guía completa de la homosexualidad. Madrid: Nuer Ediciones.

Gorer, G. (1969). Vida e ideas del Marqués de Sade. Buenos Aires: La Pleyade.

Haywood, J. (2002). Atlas histórico del mundo. Alemania: Könemann.

Hunt, L. (2005). "La vida privada durante la Revolución Francesa" en Ariès, P., y Duby, G. (directores). Historia de la vida privada. Tomo 4. De la Revolución Francesa a la Primera Guerra Mundial. Madrid: Taurus. 
Kant, I. (1932). Lo bello y lo sublime. Ensayo de estética y moral. Madrid: Espasa-Calpe. Madrid.

Laks-Eizirik, C. (2004). "Contexto histórico cultural de lo masculino y de lo femenino" en Alizade, A., Silveira, M., y Gus, M. (Compiladores). Masculino-Femenino. Cuestiones psicoanalíticas contemporáneas. Buenos Aires: Lumen.

Molina-Petit, C. (1994). Dialéctica feminista de la Ilustración. Barcelona: Anthropos.

Pasolini, P. P. (Director) (1975). Saló o le 120 giornate di Sodoma [Video, película]. Producción de Les Productions Artistes Associés y Produzioni Europee Associati (PEA). [Basada en la obra del Marqués de Sade, Las 120 jornadas de Sodoma. Ver referencia bibliográfica]

Pateman, C. (1996). "Críticas feministas a la dicotomía público/privado" en Castells, C. (Compiladora). Perspectivas feministas en teoría política. Barcelona: Paidós.

Puleo, A. H. (1992). Dialéctica de la sexualidad. Género y sexo en la filosofía contemporánea. Madrid: Cátedra.

Rich, A. (1999). "La heterosexualidad obligatoria y la existencia lesbiana" en Navarro, M., y Stimpson, C. Sexualidad, género y roles. México: Fondo de Cultura Económica.

Roudinesco, É. (2009). Nuestro lado oscuro. Una historia de los perversos. Barcelona: Anagrama.

Rousseau, J. J. (2001). Discurso sobre el origen de la desigualdad entre los hombres. Madrid: ALBAS.

Rousseau, J. J. (1985). Emilio. Madrid: EDAF.

Sade, D. A. F., Marqués de (2011). Cuentos, Historietas y Fábulas. Madrid: EDIMAT.

Sade, D. A. F., Marqués de (2009a). Juliette o Las prosperidades del vicio. Barcelona: Tusquets.

Sade, D. A. F., Marqués de (2009b). La Marquesa de Gange. Madrid: EDIMAT.

Sade, D. A. F., Marqués de (2009c). Las 120 Jornadas de Sodoma. Buenos Aires: Gradifco.

Sade, D. A. F., Marqués de (2008a). Justine o Los infortunios de la virtud. Barcelona: Tusquets.

Sade, D. A. F., Marqués de (2008b). Los crímenes del amor. Novelas heroicas y trágicas precedidas de una "Idea sobre las novelas". Madrid: Valdemar.

Sade, D. A. F., Marqués de (2003a). Filosofía en el tocador. Madrid: Mestas ediciones.

Sade, D. A. F., Marqués de (2003b). La Nueva Justine o Las desgracias de la virtud. Madrid: Valdemar. 
Sade, D. A. F., Marqués de (2003c). The Charenton Journals. The Ghosts of Sodom. [Sin lugar] Creation Books. (También contiene: Notes for "The Days at Florbelle"; "Charenton Letters"; y "Last Will and Testament”. Introducción (y traducción) por John Phillips).

Sade, D. A. F., Marqués de (1995a). La Verdad. La Vérité. Buenos Aires: Atuel-Anáfora.

Sade, D. A. F., Marqués de (1995b). Los infortunios de la virtud. Barcelona: Edicomunicación.

Sade, D. A. F., Marqués de (1980). Diálogo entre un sacerdote y un moribundo. Barcelona: Argonauta.

Sade, D. A. F., Marqués de (1969). Escritos políticos. Buenos Aires: Quintaria.

Sade, D. A. F., Marqués de (1954). Adelaide of Brunswick. Washington, D. C.: The Scarecrow Press.

Seifert, M. (2012). "Lazos de familia en la Revolución. Tensiones y cruces entre lo público y lo privado en Filosofía en el Tocador del Marqués de Sade y en Noventa y Tres de Víctor Hugo" en Hápax. Revista de la Sociedad de Estudios de Lengua y Literatura, No.5, p. 1127.

\section{¿Cómo citar este artículo?}

Solano-Fallas, A. (2017). Marqués de Sade: en contra del sexo-género determinista y la heterosexualidad excluyente. Revista humanidades, 7(2). doi:

http://dx.doi.org/10.15517/h.v7i2.29577 